\title{
Tensile fracture analysis of blunt notched PMMA specimens by means of the Strain Energy Density
}

\author{
A. Campagnolo and F. Berto*
}

\begin{tabular}{l} 
University of Padova, Department of M \\
\hline A R T I C L E I N F O \\
\hline Article history: \\
Received September 6, 2014 \\
Accepted 12 November 2014 \\
Available online \\
12 December 2014 \\
\hline Keywords: \\
Polymethylmethacrylate \\
U-notch \\
V-notch \\
Static \\
Elasticity \\
Strain energy
\end{tabular}

agement and Engineering, Stradella S. Nicola 3, 36100 Vicenza, Italy

\section{A B S T R A C T}

\begin{abstract}
In this paper, a volume criterion based on a simple scalar quantity, the mean value of the strain energy (SED), has been used to assess the static strength of notched components made of Polymethylmethacrylate (PMMA). The local-strain-energy based approach has been applied to a well-documented set of experimental data recently reported in the literature. Data refer to blunt U-notched cylindrical specimens of commercial PMMA subjected to static loads and characterised by a large variability of notch tip radius (from $0.67 \mathrm{~mm}$ to $2.20 \mathrm{~mm}$ ). Critical loads obtained experimentally have been compared with the theoretical ones, estimated by keeping constant the mean value of the strain energy in a well-defined small size volume. In addition, some new tests dealing with V-notched specimens with end holes have been carried out to investigate the effect of the notch opening angle.
\end{abstract}

(C) 2015 Growing Science Ltd. All rights reserved.

\section{Introduction}

When we want to assess the strength of notched components, both under fatigue and static loads, there is the necessity of introducing a material-dependent parameter, which typically has units of length. In the literature there are different group of methodologies. The Cohesive Zone Model (CZM) is one of the most important approaches. It was first proposed for concrete and later successfully extended to brittle or quasi-brittle failure of a large bulk of materials and in particular PMMA specimens (Gomez et al., 2000; Elices et al., 2002; Gomez et al., 2005). Another useful failure criteria, employed for analysing theoretically the brittle fracture in cracked or notched members, is the maximum tangential stress (MTS) approach (Ayatollahi et al., 2006, 2011; Ayatollahi and Aliha 2009; Torabi, 2013a, b,c; Mirsayar and Samaei, 2014).

A coherent theory of fracture that can consistently account for the fracture behaviour of both sharp cracks and blunt notches was proposed by Sih (1974) by applying the strain energy density (SED) criterion to the bulk of the material near the notch surface. The parameter $S$ is the product of the strain

* Corresponding author.

E-mail addresses: aberto@gest.unipd.it (F. Berto ) 
energy density and a small distance from the point of singularity. Failure was thought of as controlled by a critical value of $S$, whereas the direction of crack propagation is determined by imposing a minimum condition on $S$. Different from Sih's criterion, which is a point-wise criterion, the averaged strain energy density criterion (SED) states that brittle failure occurs when the mean value of the strain energy density over a control volume is equal to a critical energy (Lazzarin \& Zambardi, 2001; Lazzarin \& Berto, 2005; Berto \& Barati, 2011; Lazzarin et al., 2014). For a review of the SED approach the reader can refer to some recent papers (Berto \& Lazzarin, 2009; Lazzarin \& Berto, 2014). An important advantage of the mean SED approach with respect to the stress based criteria is the mesh independency (Lazzarin et al., 2010). The latest developments and applications have been presented in (Pook et al., 2014; Campagnolo et al., 2015) by highlighting the capacity to automatically take into account also three-dimensional effects.

The approach will be applied here to a number of experimental data taken from the literature (Zheng et al., 2003) and obtained from static tests carried out on PMMA cylindrical specimens, weakened by blunt U-notches. The experimental values of the critical load are compared with the theoretical predictions based on SED showing a sound agreement. Finally to consider the effect of the notch opening angle some tests have been carried out by the present authors (Berto et al., 2013) on symmetric $\mathrm{V}$-notches with end holes. The final assessment based on SED shows a good agreement between experimental results and theoretical values.

\section{Analytical Frame: strain energy density for blunt V-notches under mode I loading}

The SED approach is based on the idea that under tensile stresses failure occurs when the averaged value of the strain energy over a control volume (area in plane models) $\bar{W}$ reaches the critical value $W_{\mathrm{c}}$, which obviously varies from material to material. If the material behaviour is ideally brittle, then $W_{\mathrm{c}}$ can be evaluated by using simply the conventional ultimate tensile strength $\sigma_{\mathrm{t}}$, so that $W_{\mathrm{c}}=\sigma_{\mathrm{t}}{ }^{2} / 2 E$, where $E$ is the modulus of elasticity. In the case of blunt notches, the area assumes a crescent shape, with $R_{\mathrm{c}}$ being its maximum width as measured along the notch bisector line (Fig. 1c). By using the elastic maximum notch stress $\sigma_{\max }$, it is possible to determine the mean value of the SED (Lazzarin \& Berto, 2005). Under plane strain conditions, a useful expression for $R_{\mathrm{c}}$ has been provided considering the crack case (Lazzarin \& Zambardi, 2001; Lazzarin \& Berto, 2005):

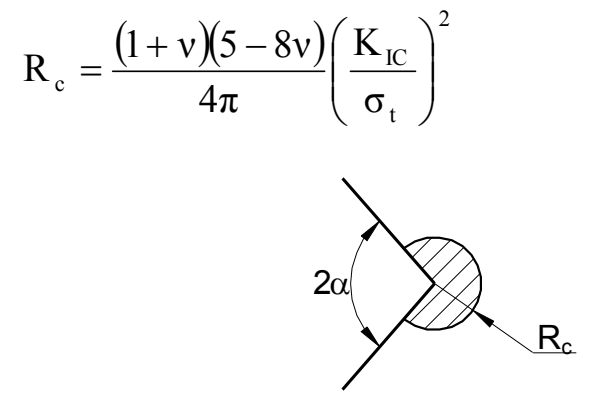

(a)

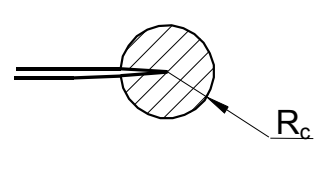

(b)

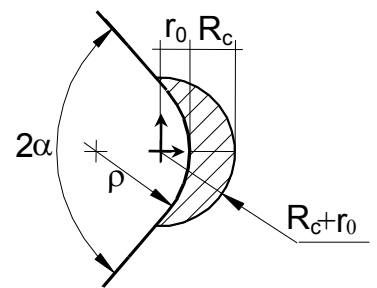

(c)

Fig. 1. Critical volume definition for sharp V-notch (a), crack (b) and blunt V-notch (c).

\section{Experimental data}

The SED-approach is applied here to a set of data that refers to circumferentially U-notched roundrod specimens tested under tensile loading, made of a commercial un-oriented PMMA (Zheng et al., 2003). The specimens were characterized by three values of the notch tip radius $\rho=0.67,1.32$ and 2.20 $\mathrm{mm}$. The material was characterised by a Poisson's ratio $v=0.4$, a modulus of elasticity $E=3.20 \mathrm{GPa}$ and a fracture toughness $K_{\mathrm{IC}} \approx 1.00 \mathrm{MPa} \mathrm{m}^{0.5}$. The value of the linear elastic 'critical tensile stress' at 
the notch root was $76.00 \mathrm{MPa}$, while the critical strain energy $W_{\mathrm{c}}$ was $0.90 \mathrm{MJ} / \mathrm{m}^{3}$, respectively. The stress-strain curve showed a linear elastic trend up to failure.

To consider also the effect of the notch opening angle some new tests have been carried out by the present authors on double symmetric V-notched specimens with end holes characterized by a notch opening angle equal to $30^{\circ}$ and $120^{\circ}$. The geometry of the specimens is shown in Fig. 2. Three different notch root radii were used in the experiments: $\rho=0.5,1$ and $2 \mathrm{~mm}$.
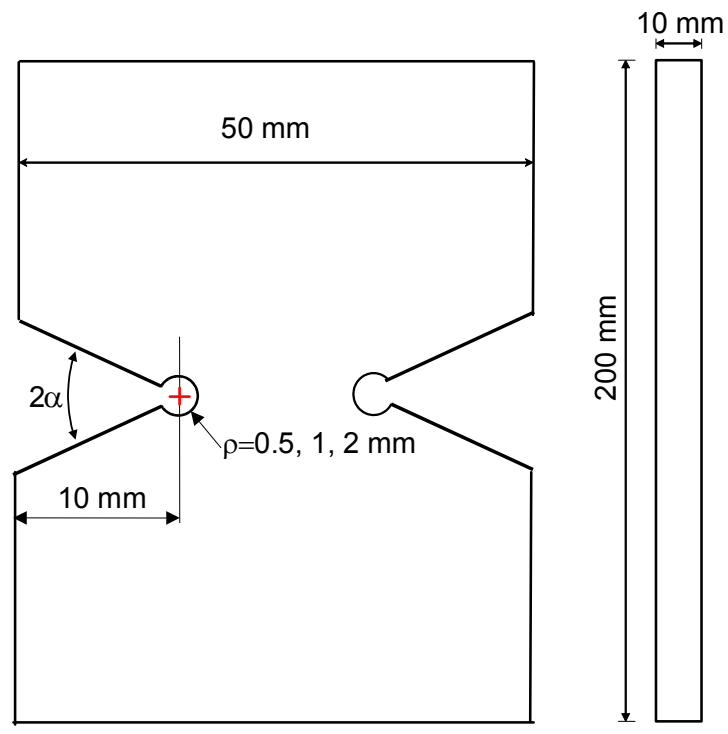

Fig. 2. Geometry of the PMMA specimens weakened by V-notches with end holes.

Being the PMMA the same used in (Gomez et al., 2007), the critical stress has been confirmed equal to $130 \mathrm{MPa}$ while the critical energy equal to $W_{\mathrm{c}}=1.632 \mathrm{MJ} / \mathrm{m}^{3}$. All tests were performed at room temperature under displacement control on a servocontrolled MTS bi-axial testing device $( \pm 100 \mathrm{kN} / \pm$ $110 \mathrm{Nm}, \pm 75 \mathrm{~mm} / \pm 55^{\circ}$ ). The load was measured by a MTS cell with $\pm 0.5 \%$ error at full scale.

\section{Application of the SED approach}

Finite element (FE) modelling was performed using ANSYS 12.1 in order to determine the mean value of the strain energy density (SED) at failure relating to the different specimens. The simulations were conducted under purely linear-elastic conditions, in a state of plane strain for the plain specimens while the axial-symmetry option was activated when considering the cylindrical specimens. The mesh was performed by using the eight nodes element (PLANE 82). By using Eq. 1 and the above mentioned properties for the material tested in (Zheng et al., 2003) and for the material tested by the present authors, $R_{\mathrm{c}}$ results to be $0.035 \mathrm{~mm}$ in both cases. In order to evaluate the mean value of the strain energy density at failure, the critical loads obtained experimentally on the tested specimens have been directly applied to the FE-models.

\section{Results and discussion}

A synthesis in terms of the square root value of the local energy at failure, averaged over the control volume (of radius $R_{\mathrm{c}}$ ), obtained by FE-analysis and normalised with respect to the critical energy of the 
material as a function of the notch tip radius $\rho$ is shown in Fig. 3 and 4 for the new data. The plotted parameter is proportional to the fracture load. All the values fall inside a scatter ranging from 0.8 to 1.2 and this states true for both the kind of PMMA considered in the present investigation. Then it is possible to consider the averaged strain energy density (SED) at failure as constant and equal to the critical value characteristic of the material.

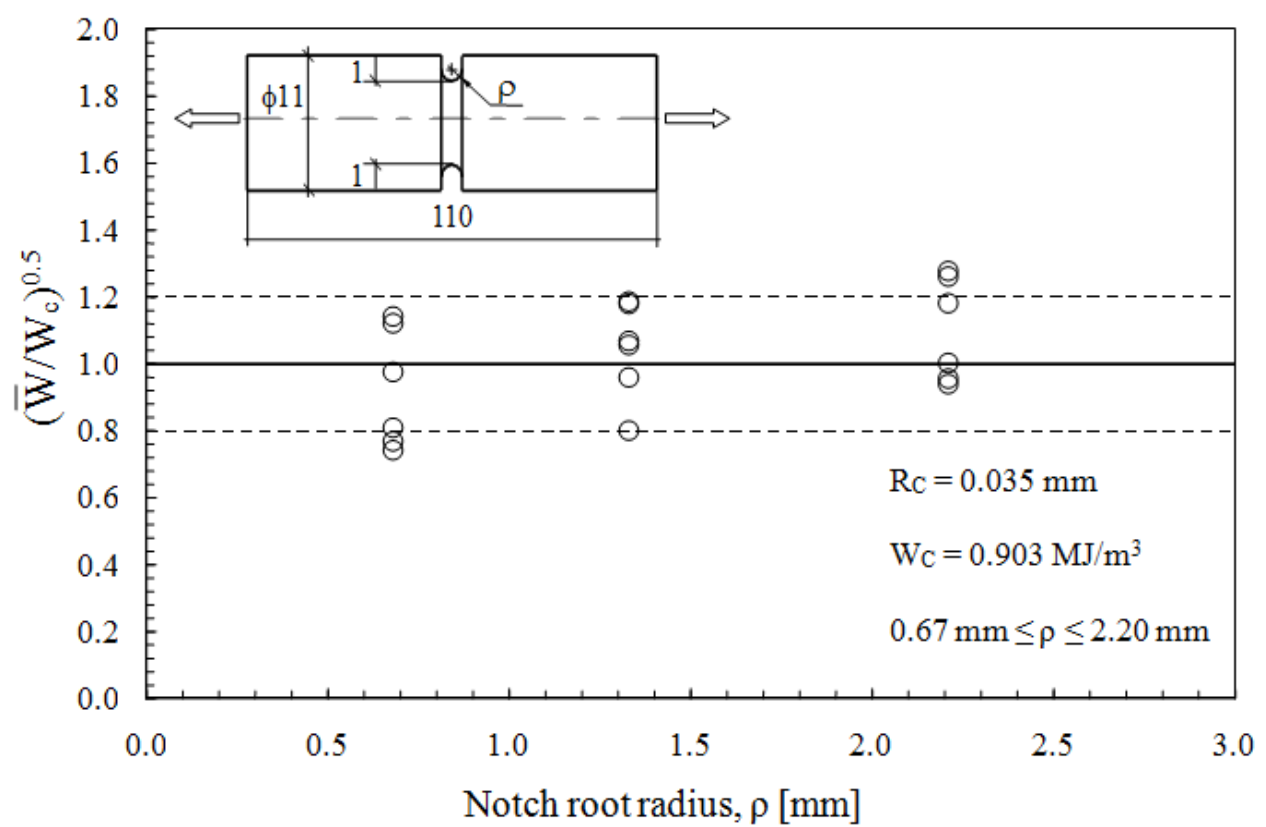

Fig. 3. Scatter of the strain energy density values at failure for circumferentially U-notched round-rod specimens made of PMMA (Zheng et al., 2003)

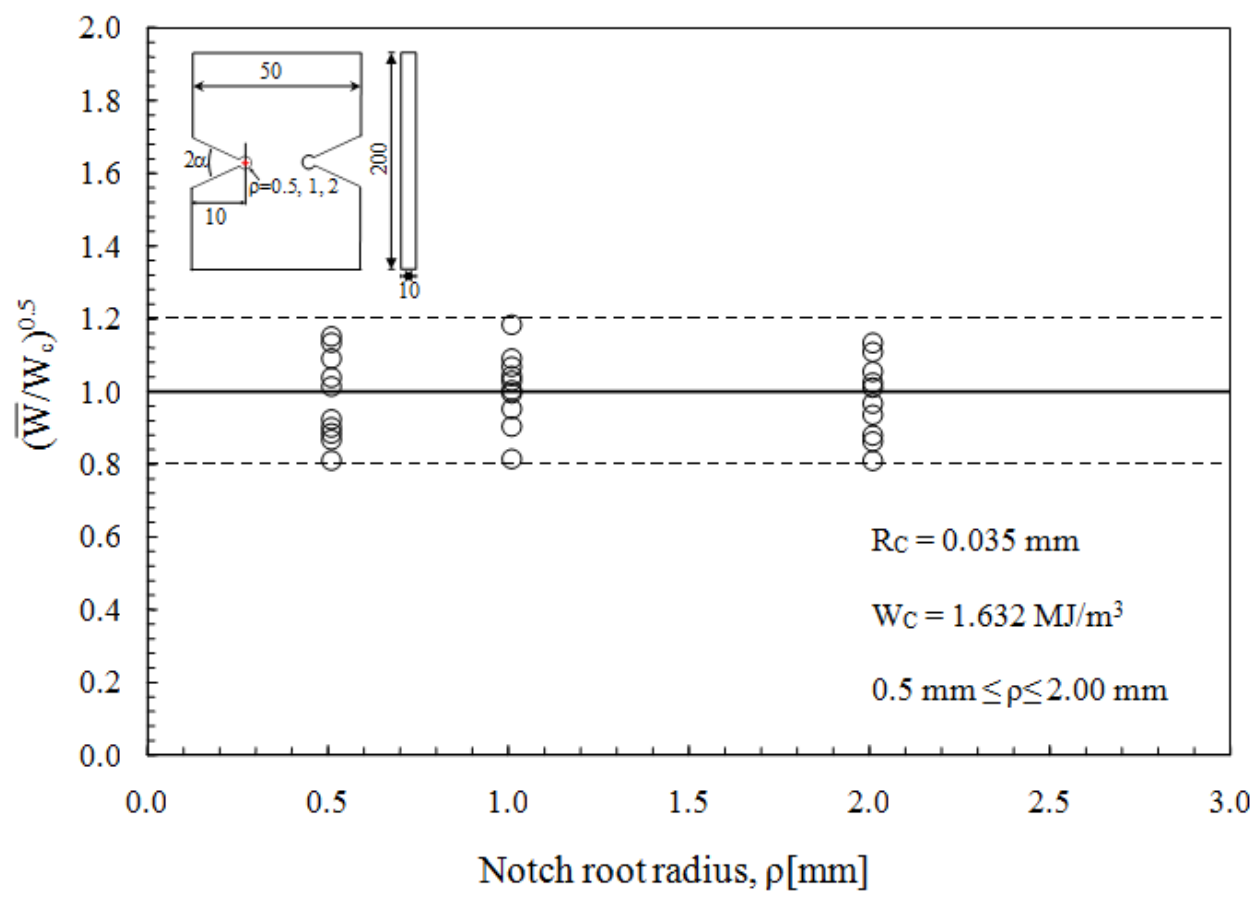

Fig. 4. Scatter of the strain energy density values at failure for specimens weakened by symmetric Vnotches with end holes and made of PMMA

In Fig. 5 the experimental values of the critical loads (open dots) have been compared with the theoretical assessed values based on the constancy of the SED in the control volume (solid lines). As shown in the figure it is evident that the trend of the predicted loads is in good agreement with the 
experimental one, being the maximum deviation less than $20 \%$ for the data taken from Zheng et al. (2003). Table 1 reports the results of the FE-analysis, in terms of averaged SED at failure, and the direct comparison between the mean experimental loads and the theoretical assessed values.

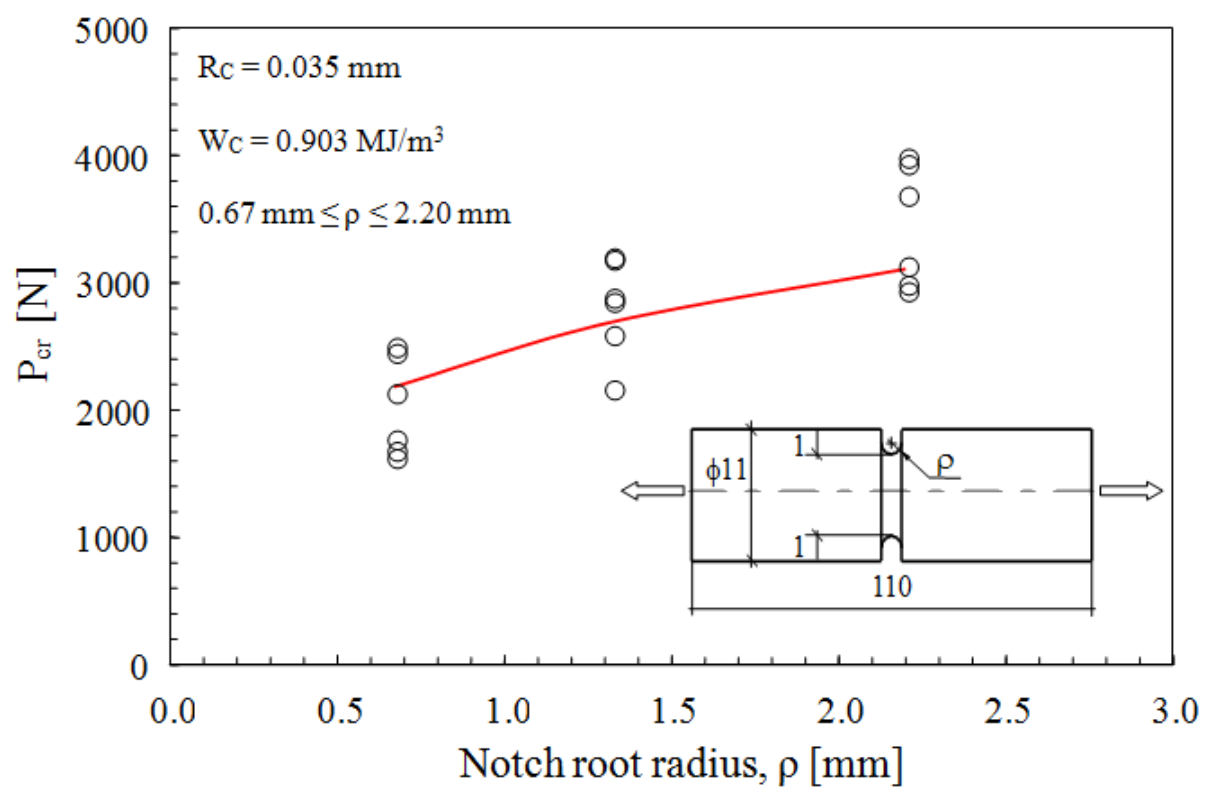

Fig. 5. Critical load $P_{\text {cr }}$ versus notch radius $\rho$, comparison between theoretical predictions and experimental data for specimens made of PMMA (Zheng et al., 2003); predictions based on constant value $\mathrm{W}_{\mathrm{c}}=0.903 \mathrm{MJ} / \mathrm{m}^{3}$

Table 1. Strain energy as obtained from numerical analyses of circumferentially U-notched round-rod specimens made of PMMA (Zheng et al., 2003). $W_{\mathrm{c}}=0.903 \mathrm{MJ} / \mathrm{m}^{3}$

\begin{tabular}{|c|c|c|c|c|c|c|c|}
\hline $\begin{array}{c}\boldsymbol{D} \\
\mathrm{mm}\end{array}$ & $\begin{array}{c}\boldsymbol{L} \\
\mathrm{mm}\end{array}$ & $\begin{array}{c}\boldsymbol{a} \\
\mathrm{mm}\end{array}$ & $\begin{array}{c}\boldsymbol{\rho} \\
\mathrm{mm}\end{array}$ & $\begin{array}{l}\boldsymbol{P} \\
\mathrm{N}\end{array}$ & $\begin{array}{c}\boldsymbol{P}_{\text {th }} \\
\mathrm{N}\end{array}$ & $\begin{array}{c}\overline{\mathbf{W}}_{1} \\
\mathrm{MJ} / \mathrm{m}^{3}\end{array}$ & $\left(\overline{\mathbf{W}}_{1} / \mathbf{W}_{\mathrm{C}}\right)^{0.5}$ \\
\hline \multirow{6}{*}{11} & \multirow{6}{*}{110} & \multirow{6}{*}{1} & 0.67 & 1641.33 & 2180.78 & 0.512 & 0.753 \\
\hline & & & 0.67 & 1698.58 & & 0.548 & 0.779 \\
\hline & & & 0.67 & 1787.64 & & 0.607 & 0.820 \\
\hline & & & 0.67 & 2150.26 & & 0.878 & 0.986 \\
\hline & & & 0.67 & 2468.35 & & 1.157 & 1.132 \\
\hline & & & 0.67 & 2512.88 & & 1.199 & 1.152 \\
\hline \multirow{6}{*}{11} & \multirow{6}{*}{110} & \multirow{6}{*}{1} & 1.32 & 2182.07 & 2689.63 & 0.594 & 0.811 \\
\hline & & & 1.32 & 2608.31 & & 0.849 & 0.970 \\
\hline & & & 1.32 & 2869.14 & & 1.028 & 1.067 \\
\hline & & & 1.32 & 2900.95 & & 1.050 & 1.079 \\
\hline & & & 1.32 & 3199.95 & & 1.278 & 1.190 \\
\hline & & & 1.32 & 3219.03 & & 1.293 & 1.197 \\
\hline \multirow{6}{*}{11} & \multirow{6}{*}{110} & \multirow{6}{*}{1} & 2.20 & 2951.84 & 3107.33 & 0.815 & 0.950 \\
\hline & & & 2.20 & 3002.73 & & 0.843 & 0.966 \\
\hline & & & 2.20 & 3149.05 & & 0.927 & 1.013 \\
\hline & & & 2.20 & 3702.52 & & 1.282 & 1.192 \\
\hline & & & 2.20 & 3950.63 & & 1.460 & 1.271 \\
\hline & & & 2.20 & 4001.53 & & 1.497 & 1.288 \\
\hline
\end{tabular}

Dealing with the new data, Fig. 6 shows the experimental values of the critical loads compared with the theoretical one, in the case of a notch opening angle equal to $30^{\circ}$. The relative deviation between 
experimental and theoretical values is always lower than $15 \%$, in good agreement with previous tests under mode I loading (Lazzarin and Berto, 2005). Table 2 reports the results of the FE-analysis, in terms of averaged SED at failure, and the direct comparison between the mean experimental loads and the theoretical assessed values, for the new data.
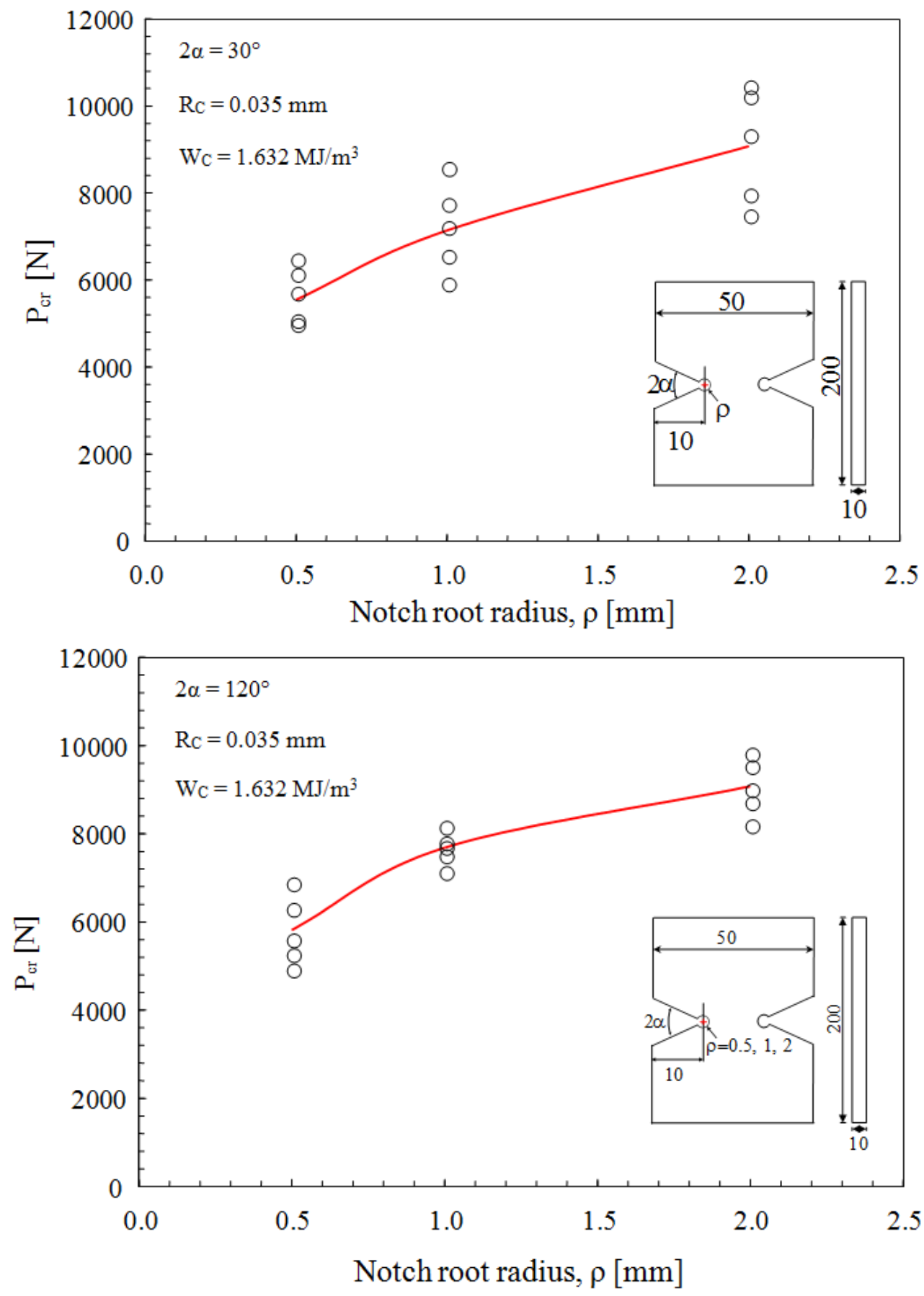

Fig. 6. Critical load $P_{\text {cr }}$ versus notch radius $\rho$, comparison between theoretical predictions and experimental data for specimens weakened by symmetric V-notches with end holes and made of PMMA; predictions based on constant value $\mathrm{W}_{\mathrm{c}}=1.632 \mathrm{MJ} / \mathrm{m}^{3} ; 2 \alpha=30^{\circ}$ (a) $2 \alpha$ $=120^{\circ}(\mathrm{b})$ 
Table 2. Strain energy as obtained from numerical analyses of specimens weakened by symmetric Vnotches with end holes and made of PMMA, $W_{\mathrm{c}}=1.632 \mathrm{MJ} / \mathrm{m}^{3}$

\begin{tabular}{ccccccccc}
\hline $\begin{array}{c}\boldsymbol{\alpha} \boldsymbol{\alpha} \\
\circ\end{array}$ & $\boldsymbol{W}$ & $\boldsymbol{B}$ & $\boldsymbol{a}$ & $\boldsymbol{\rho}$ & $<\boldsymbol{P}>$ & $\boldsymbol{P}_{\text {th }}$ & $\overline{\mathbf{W}}_{\mathbf{1}}$ & $\left(\overline{\mathbf{W}}_{\mathbf{1}} / \mathbf{W}_{\mathbf{C}}\right)^{\mathbf{0 . 5}}$ \\
\hline 30 & $\mathrm{~mm}$ & $\mathrm{~mm}$ & $\mathrm{~mm}$ & $\mathrm{~mm}$ & $\mathrm{~N}$ & $\mathrm{~N}$ & $\mathrm{MJ} / \mathrm{m}^{3}$ & \\
\hline 30 & 50 & 10 & 10 & 0.5 & 5699 & 5546 & 1.707 & 1.017 \\
30 & 50 & 10 & 10 & 2 & 9114 & 9074 & 1.642 & 0.994 \\
\hline 120 & 50 & 10 & 10 & 0.5 & 5816 & 5973 & 1.539 & 0.964 \\
120 & 50 & 10 & 10 & 1 & 7682 & 7366 & 1.744 & 1.033 \\
120 & 50 & 10 & 10 & 2 & 9076 & 9155 & 1.579 & 0.982 \\
\hline
\end{tabular}

\section{Conclusions}

An approach, useful for the assessment of the strength of notched components and based on the mean value of the strain energy over a small size area surrounding the tip of the notches, has been applied here to a number of experimental data taken from the literature and obtained from static tests carried out on PMMA cylindrical specimens, weakened by blunt U-notches. In addition some new data from PMMA V-notched specimens with end holes are reported in the paper.

The results of the synthesis based on SED show that the averaged values of the strain energy density at failure can be considered as a constant for the material and equal to the critical value, $W_{\mathrm{c}}$. The experimental loads can be assessed with a maximum error of $\pm 20 \%$ which in many cases remains in the range of $\pm 10 \%$.

\section{References}

Ayatollahi, M. R., Aliha, M. R. M., \& Hassani, M. M. (2006). Mixed mode brittle fracture in PMMAan experimental study using SCB specimens. Materials Science and Engineering: A, 417(1), 348356.

Ayatollahi, M. R., \& Aliha, M. R. M. (2009). Analysis of a new specimen for mixed mode fracture tests on brittle materials. Engineering Fracture Mechanics, 76(11), 1563-1573.

Ayatollahi, M. R., Aliha, M. R. M., \& Saghafi, H. (2011). An improved semi-circular bend specimen for investigating mixed mode brittle fracture. Engineering Fracture Mechanics, 78(1), 110-123.

Berto, F., \& Lazzarin, P. (2009). A review of the volume-based strain energy density approach applied to V-notches and welded structures. Theoretical and Applied Fracture Mechanics, 52(3), 183-194.

Berto, F., \& Barati, E. (2011). Fracture assessment of U-notches under three point bending by means of local energy density. Materials \& Design, 32(2), 822-830.

Berto, F., \& Lazzarin, P. (2014). Recent developments in brittle and quasi-brittle failure assessment of engineering materials by means of local approaches. Materials Science and Engineering: $R$ : Reports, 75, 1-48.

Berto, F., Campagnolo, A., Elices, M., \& Lazzarin, P. (2013). A synthesis of Polymethylmethacrylate data from U-notched specimens and $\mathrm{V}$-notches with end holes by means of local energy. Materials \& Design, 49, 826-833.

Campagnolo, A., Berto, F., \& Lazzarin, P. (2015). The effects of different boundary conditions on three-dimensional cracked discs under anti-plane loading. European Journal of Mechanics-A/Solids. , 50, 76-86.

Elices, M., Guinea, G. V., Gomez, J., \& Planas, J. (2002). The cohesive zone model: advantages, limitations and challenges. Engineering fracture mechanics, 69(2), 137-163. 
Gómez, F. J., Elices, M., \& Valiente, A. (2000). Cracking in PMMA containing U-shaped notches. Fatigue \& Fracture of Engineering Materials \& Structures, 23(9), 795-803.

Gómez, F. J., Elices, M., \& Planas, J. (2005). The cohesive crack concept: application to PMMA at60 C. Engineering fracture mechanics, 72(8), 1268-1285.

Gómez, F. J., Elices, M., Berto, F., \& Lazzarin, P. (2007). Local strain energy to assess the static failure of U-notches in plates under mixed mode loading. International Journal of Fracture, 145(1), 29-45.

Mirsayar, M., \& Samaei, A. (2014). Application of maximum tangential stress criterion in determination of fracture initiation angles of silicon/glass anodic bonds. Engineering Solid Mechanics, 2(3), 145-150.

Lazzarin, P., \& Zambardi, R. (2001). A finite-volume-energy based approach to predict the static and fatigue behavior of components with sharp V-shaped notches. International Journal of Fracture, 112(3), 275-298.

Lazzarin, P., \& Berto, F. (2005). Some expressions for the strain energy in a finite volume surrounding the root of blunt V-notches. International Journal of Fracture, 135(1-4), 161-185.

Lazzarin, P., Berto, F., \& Zappalorto, M. (2010). Rapid calculations of notch stress intensity factors based on averaged strain energy density from coarse meshes: theoretical bases and applications. International Journal of Fatigue,32(10), 1559-1567.

Lazzarin, P., Campagnolo, A., \& Berto, F. (2014). A comparison among some recent energy-and stressbased criteria for the fracture assessment of sharp V-notched components under Mode I loading. Theoretical and Applied Fracture Mechanics. 71, 21-30.

Pook, L. P., Berto, F., Campagnolo, A., \& Lazzarin, P. (2014). Coupled fracture mode of a cracked disc under anti-plane loading. Engineering Fracture Mechanics, 128, 22-36.

Sih, G. C. (1974). Strain-energy-density factor applied to mixed mode crack problems. International Journal of fracture, 10(3), 305-321.

Torabi, A. (2013 a). Wide range brittle fracture curves for U-notched components based on UMTS model. Engineering Solid Mechanics, 1(2), 57-68.

Torabi, A. (2013 b). Failure curves for predicting brittle fracture in V-notched structural components loaded under mixed tension/shear: An advanced engineering design package. Engineering Solid Mechanics, 1(3), 99-118.

Torabi, A. (2013 c). The equivalent material concept: application to failure of O-notches. Engineering Solid Mechanics, 1(4), 129-140.

Zheng, X. L., Wang, H., \& Yan, J. H. (2003). Notch strength and notch sensitivity of polymethyl methacrylate glasses. Materials Science and Engineering: A, 349(1), 80-88. 